\title{
Does the Counterfactual Theory of
}

\author{
Explanation Apply to Non-Causal
}

\section{Explanations in Metaphysics?}

\author{
Alexander Reutlinger \\ Munich Center for Mathematical Philosophy \\ LMU Munich \\ Alexander.Reutlinger@lmu.de
}

In the recent philosophy of explanation, a growing attention to and discussion of non-causal explanations has emerged, as there seem to be compelling examples of non-causal explanations in the sciences, in pure mathematics, and in metaphysics. I defend the claim that the counterfactual theory of explanation (CTE) captures the explanatory character of both non-causal scientific and metaphysical explanations. According to the CTE, scientific and metaphysical explanations are explanatory by virtue of revealing counterfactual dependencies between the explanandum and the explanans. I support this claim by illustrating that CTE is applicable to Euler's explanation (an example of a non-causal scientific explanation) and Loewer's explanation (an example of a non-causal metaphysical explanation). 


\section{Introduction}

Since the early 2000 s, one finds a strikingly common theme in philosophy of science, philosophy of mathematics, and metaphysics: an increasing attention to non-causal explanations. ${ }^{1}$ Many philosophers of science, philosophers of mathematics and metaphysicians take seriously the view that (a) there are alongside with familiar causal explanations - compelling examples of non-causal explanations in the sciences, and that (b) there are instances of non-causal explanations in pure mathematics and in metaphysics. According to this view, explanation is a goal of many epistemic endeavors, not merely of the natural and social sciences.

A significant amount of work has been dedicated to understanding the differences and commonalities of explanations in the sciences and in pure mathematics (see Steiner 1978; Kitcher 1984; and, more recently, Mancosu 2011; Lange 2013, 2014). However, the debates on scientific explanation and metaphysical explanation are, by and large, not well connected (Ruben 2012; Schaffer 2015, and Wilson [manuscript] are notable recent exceptions). In this paper, I will only attempt to make a connection between two of these largely separate debates in arguing that at least some non-causal explanations in the sciences and in metaphysics can be captured by the same theory of explanationthe counterfactual theory of explanation. Due to the complexity of the subject matter, I will not address non-causal explanations in pure mathematics in this paper.

\footnotetext{
${ }^{1}$ This attention to non-causal explanations sometimes takes a critical tone - see, for instance, Bueno and French (2012), Skow (2014), and Strevens (forthcoming). However, it is not the goal of this paper to discuss the critics of (certain kinds of) non-causal explanations.
} 
What motivates the increasing attention to non-causal explanations? In the recent literature, the view that one finds non-causal explanations in the sciences, pure mathematics, and metaphysics is supported by the following examples:

- Non-causal (and causal) explanations in the sciences. Without doubt, there are well-known examples of causal explanations in the natural and social sciences, including detailed mechanistic explanations (Bechtel and Richardson 1993; Machamer et al. 2000) and 'higher-level', 'coarse-grained', or 'macro' causal explanations (Cartwright 1989; Woodward 2003). However, the causal explanations do not exhaust the class of all scientific explanations, or so several philosophers have argued. In addition to causal explanations, one encounters compelling examples of non-causal explanations. Such examples include different kinds of so-called 'mathematical' explanations of contingent empirical phenomena (not to be confused with explanations in pure mathematics!) such as graph-theoretic (Pincock 2012; Lange 2013a; see already van Fraassen 1989), topological (Huneman 2010; Lange 2013a), geometric (Lange 2013a), and genuinely statistical explanations (Lipton 2004; Lange 2013b). For the sake of convenience, I will call these explanations 'applied mathematical' explanations. Other kinds of non-causal explanations in physics include explanations based on symmetry principles and conservation laws (Lange 2011), kinematics (Saatsi forthcoming), renormalization group theory (Batterman 2000, Reutlinger forthcoming), dimensional analysis (Lange 2009a, Pexton 2014), and inter-theoretic relations (Batterman 2002; Weatherall 2011). ${ }^{2}$

\footnotetext{
${ }^{2}$ Explanations based on network models are promising, though controversial,
} 
- Non-causal explanations in pure mathematics. Philosophers of mathematics and mathematicians distinguish between explanatory and non-explanatory proofs, where the former are taken to be explanatory in a non-causal way (see, for instance, in the recent literature, Mancosu 2008, 2011; and Lange 2014; see Steiner 1978; Kitcher 1984, 1989 for pioneering work). The explananda of explanatory proofs in pure mathematics are theorems, while applied mathematical explanations explain the occurrence of contingent phenomena.

- Non-causal explanations in metaphysics. A growing number of metaphysicians adopt the view that if one kind of facts grounds another kind of facts, then the former facts metaphysically explain why the latter kind of facts exists. ${ }^{3}$ For instance, a physicalist holds that if physical facts ground mental facts, then the physical facts metaphysically explain the existence of the mental facts. In the current debates in metaphysics, grounding is taken to be a non-causal dependence relation whose explanatory power is, thus, noncausal (to take only a few voices from the enormous literature on grounding, see Fine 2001, 2012; Schaffer 2009, 2015; Schnieder 2011; Correira and Schnieder 2012a; Loewer 2012; Ruben 2012; Wilson manuscript).

The view that there are non-causal explanations in the sciences, in pure mathematics, and in metaphysics raises an interesting challenge, because this view is, prima facie, in tension with the currently dominating causal accounts of explanation in philosophy of science and elsewhere. According to causal accounts, there is a tight conceptual connection between explaining and candidates for non-causal explanations in the life and social sciences (Strogatz 1994; Barabasi et al. 2006; Huneman 2010).

${ }^{3}$ Note that grounding need not be restricted to facts. It can be extended to other kinds of entities (see Fine 2001, 2012; Schaffer 2009). 
identifying or representing causes (see, among many others, Salmon 1984; Cartwright 1989; Machamer et al. 2000; Woodward 2003; Strevens 2008). The common core of seminal causal accounts of explanation can be expressed as follows: to explain some phenomenon P just is to identify the (type or token level) causes of P. Examples of non-causal explanations are a direct challenge to causal accounts, because causal accounts of explanation - prima facie - cannot accommodate non-causal explanations and, hence, causal accounts do not provide a general account of all scientific explanations (as van Fraassen [1980: 123]; Achinstein [1983: 230-243]; Lipton [2004: 32] already noted). This dialectic situation leaves us with the task to come up with an appropriate theoretical response to the apparent fact that there are not only causal but also non-causal explanations. (However, note that Lewis 1986 and Skow 2014 advocate a significantly weakened, alternative causal account of explanation, see footnote 4 below.)

In this paper, I will defend one specific monist approach to explanation, a counterfactual theory of explanation. I take monism to be the view that there is one single philosophical account capturing both causal and non-causal explanations. A monist holds that causal and non-causal explanations share a feature that makes them explanatory. Hempel's covering-law account is an instructive historical example for illustrating monism. Hempel argued that causal and non-causal explanations are explanatory by virtue of having one single feature in common: nomic expectability (Hempel 1965: 352). In the case of causal explanations, one expects the explanandum to occur on the basis on causal covering laws (laws of succession) and initial conditions; in the non-causal case, one's expectations are based on non-causal covering laws (laws of coexistence) 
and initial conditions. Moreover, in order to be applicable to mathematical and metaphysical explanations, a friendly amendment of the covering law account may even allow for (a) metaphysical covering laws (such as general statements about one kind of facts grounding another kind of facts), and (b) mathematical covering laws (that is, mathematical axioms). However, due to well-known problems of the covering-law account (Salmon 1989: 46-50), Hempel's monism is at least not a viable option for dealing with all explanations in the scientific domain.

My goal in this paper is to explore a monist account that does not collapse into Hempel's troubled version of monism. I claim that a counterfactual theory of explanation is a promising candidate for playing this role (building on and elaborating recent work by Frisch 1998; Bokulich 2008; Saatsi and Pexton 2013; Reutlinger forthcoming). ${ }^{4}$ However, I do not want to argue for a full-fledged monism, i.e. the claim that the counterfactual theory captures all kinds of (causal and) non-causal explanations in science and metaphysics (including the ones listed above). My goal in this paper is more modest: I will argue that the counterfactual theory can be successfully applied to two paradigmatic examples of non-causal explanations from science and metaphysics, which I take to be representative of a larger class of non-causal explanations: Euler's explanation (a toy example of a non-causal scientific explanation) and Loewer's explanation (an example of a non-causal metaphysical explanation). ${ }^{5}$ As indicated above, I will

\footnotetext{
${ }_{5}^{4}$ See Lange (2009b) for a related account.

${ }^{5}$ One alternative option to monism is causal reductionism, i.e. the view that seemingly non-causal explanations can ultimately be understood as causal explanations. Lewis (1986) and Skow (2014) have presented the most compelling attempt to spell out this strategy. Lewis and Skow rely on the notion of providing information about the causal history of the explanandum. Their notion of 'causal information' is significantly broader, and weaker, than the notion of 'identifying
} 
not address the issue of whether the CTE is applicable to explanations in pure mathematics in this paper.

The plan of the paper is as follows: in section 2, I introduce the counterfactual theory of explanation. In section 3 , I argue that the counterfactual theory can be successfully applied to Euler's explanation - the main purpose of this section (and of using Euler's explanation) is to illustrate how the CTE works for the (toy) case of a non-causal explanation in the sciences. In section 4, I will explore whether the CTE also applies to Loewer's explanation. In section 5, I qualify the CTE in three respects.

\section{The Counterfactual Theory of Explanation}

Is there a monist alternative to Hempel's troubled monism? It is fruitful to take a suggestion of Peter Lipton's as a stepping-stone for developing such a monist account. Having presented several examples of non-causal explanations, Lipton outlines a monist strategy for capturing both non-causal and causal explanations (in science): "One reaction to this would be to attempt to expand the notion of causation to some broader notion of 'determination' that would encompass the non-causal cases [...]." (Lipton 2004: 32) ${ }^{6}$ However, Lipton is skeptical as to

the causes of the explanandum' figuring in the causal accounts I have referred to earlier. For instance, Lewis and Skow hold that one explains causally by merely excluding a possible causal history of some explanandum $\mathrm{E}$, or by stating that $\mathrm{E}$ has no cause at all - while other causal accounts would not classify this sort of information as causally explanatory. I cannot enter an in-depth discussion of the merits of causal reductionism here. I take it as premise - against causal reductionism - that there actually are non-causal explanations including the examples listed above and the two examples I will discuss in Sections 3 and 4.

${ }^{6}$ See Kim (1974) and Ruben (2012) for related suggestions. 
whether one can prevent such a "broader notion of determination" from collapsing into Hempelian monism:

This approach has merit, but it will be difficult to come up with such a notion that we understand even as well as causation, without falling into the relation of deductive determination, which will expose the model to many of the objections to the deductivenomological model. (Lipton 2004: 32)

I think Lipton was too hasty in dismissing the merit of explicating "some broader notion of 'determination' that would encompass the non-causal cases" (ibid.), because such a philosophical project need not necessarily rely on the covering-law account and a "relation of deductive determination" (ibid.). Following Lipton's original suggestion, my claim is that Lipton's envisioned broader notion of determination is the notion of the counterfactual dependence (of the explanandum on the explanans), as captured by counterfactual theories of explanation (for short, CTE). Perhaps the most influential version of the $\mathrm{CTE}^{7}$ is James Woodward's (see Woodward 2003; and already Woodward $1979^{8}$ ). Woodward summarizes the CTE as follows:

An explanation ought to be such that it enables us to see what sort of difference it would have made for the explanandum if the factors cited in the explanans had been different in various possible ways.

\footnotetext{
${ }^{7}$ I adopt Woodward's (2003) terminology in calling it a counterfactual theory. ${ }^{8}$ It is interesting that Woodward (1979: 45-46) takes the CTE to be a charitable revision of the covering law account.
} 
(Woodward 2003: 11)

Explanation is a matter of exhibiting systematic patterns of counterfactuals dependence. (Woodward 2003: 191)

The CTE is prima facie appealing from a monist perspective for two reasons: first, Woodward's (2003: §5.3, §5.8) CTE avoids the notorious problems of the covering-law account (see below for a qualification of this claim). Second, although Woodward's version of the CTE - and the underlying interventionist theory of causation - is mainly intended to fit causal explanations, the core idea of the CTE provides a natural way for specifying Lipton's "broader notion of determination”. As Woodward suggests himself (but does not elaborate):

$[\mathrm{T}]$ he common element in many forms of explanation, both causal and non-causal, is that they must answer what-if-things-had-beendifferent questions. (Woodward 2003: 221).

Answering "what-if-things-had-been-different questions" amounts to revealing (or exhibiting) - in Woodward's words - what sort of difference it would have made for the explanandum if the factors cited in the explanans had been different in various possible ways. The monist proposal, according to the CTE, is that causal and non-causal explanations are explanatory by virtue of exhibiting how the explanandum counterfactually depends on the explanans (Woodward 2003: 13). Or, putting it in Lipton's terms, the notion of counterfactual dependence is the broader notion of determination that one "expands" from causal explanations 
such that it encompasses the non-causal explanations. This CTE-based monism has been explored, among others, by Frisch (1998), Bokulich (2008), Saatsi and Pexton (2013), Saatsi (forthcoming), and (AUTHOR) in the context of scientific explanations. My goal is to further elaborate and advance the CTE and to apply it to a novel example of non-causal explanations that proponents of the CTE have not yet addressed: namely, to a metaphysical explanation (Loewer's explanation).

I will start with reconstructing the CTE in a way that emphasizes the “common element" (Woodward 2003: 221) of causal and non-causal explanations - this common element, I conjecture, is not essentially tied to an interventionist approach to causation (I will return to this issue below). In this reconstruction I largely follow Woodward's (2003: 203) and Woodward and Hitchcock's (2003: 6, 18) exposition of the CTE. As a first step, the structure of an explanation has two parts: first, a statement $\mathrm{E}$ about the explanandum phenomenon; second, an explanans consisting of generalizations $\mathrm{G}_{1}, \ldots, \mathrm{G}_{\mathrm{m}}$ and auxiliary statements $\mathrm{S}_{1}$, $\ldots, \mathrm{S}_{\mathrm{n}}$. Auxiliary statements often are statements about initial or boundary conditions specifying the state of the explanandum system (as Hitchcock and Woodward highlight those statements typically assert that variables take a certain value). But the auxiliary statements may also comprise other kinds of statements useful for explanations (for instance, Nagelian bridge laws, symmetry assumptions, limit theorems, and other modeling assumptions). According to the CTE, the relationship between the explanans and the explanandum is explanatory iff the following conditions are all satisfied:

1. Veridicality condition: $\mathrm{G}_{1}, \ldots, \mathrm{G}_{\mathrm{m}}, \mathrm{S}_{1}, \ldots, \mathrm{S}_{\mathrm{n}}$, and $\mathrm{E}$ are (approximately) true or, at least, well confirmed. 
2. Implication condition: $\mathrm{G}_{1}, \ldots, \mathrm{G}_{\mathrm{m}}$ and $\mathrm{S}_{1}, \ldots, \mathrm{S}_{\mathrm{n}}$ logically entail $\mathrm{E}$ or a conditional probability $\mathrm{P}\left(\mathrm{E} \mid \mathrm{S}_{1}, \ldots, \mathrm{S}_{\mathrm{n}}\right)$ - where the conditional probability need not be 'high' in contrast to Hempel's covering-law account.

3. Dependency condition: $\mathrm{G}_{1}, \ldots, \mathrm{G}_{\mathrm{m}}$ support at least one counterfactual of the form: had $S_{1}, \ldots, S_{n}$ been different than they actually are (in at least one way deemed possible in the light of the generalizations), then $\mathrm{E}$ or the conditional probability of E would have been different as well. ${ }^{9}$

The CTE provides a monist framework for causal and non-causal explanations both kinds of explanation are explanatory because they rely on approximately true assumptions in the explanans, the explanans implies (a probability for the) explanandum, and, crucially, both kinds of explanation reveal counterfactual dependencies between the explanandum and the explanans.

\section{Illustrating the Counterfactual Theory: Euler's explanation}

I will now argue for the claim that the CTE applies to one paradigmatic example of a non-causal scientific explanations, Euler's explanation. I take Euler's explanation to be a 'toy' example of a non-causal scientific explanation. The purpose of using Euler's explanation is mainly to illustrate how the CTE applies to a non-causal explanation in the scientific domain (for an application of the CTE to 'more serious' non-causal scientific explanations, see Frisch 1998; Bokulich

\footnotetext{
${ }^{9}$ I assume that a generalization supports counterfactuals only if the generalization is non-accidentally true or lawful. I use a broad notion of laws that includes nonstrict ceteris paribus laws, such as Woodward and Hitckcock's own invariance account. However, my aim here is not to defend a particular view of laws. I want to suggest instead that the CTE is neutral with respect to alternative theories of non-accidental truth or lawhood. I take this to be a strength of the CTE.
} 
2008; Kistler 2013; Saatsi and Pexton 2014; Pexton 2014; Pincock 2015; Saatsi forthcoming; Reutlinger forthcoming a,b). Then, in section 4, I will argue that the CTE also applies to a paradigmatic non-causal explanation from metaphysics: namely, Loewer's metaphysical explanation of nomic facts.

Euler's explanation is an intuitively simple and powerful non-causal "graph-theoretical" explanation (van Fraassen 1989: 236-239; Pincock 2012: 5153; Lange 2013a: 489; Reutlinger forthcoming). I use Euler's explanation as a stand-in for graph-theoretical and network-based explanations (see Huneman 2010).

In 1736, Königsberg had four parts of town and seven bridges connecting these parts. Interestingly, no one, at that time, ever succeeded in the attempt to cross all of the bridges exactly once. This surprising fact calls for an explanation. The mathematician Leonhard Euler provided an explanation. Euler's explanation starts with representing relevant aspects of Königsberg's geography with a graph. A simplified geographical map of Königsberg in 1736 represents only the four parts of town (the two islands $\mathrm{A}$ and $\mathrm{B}$, and the two riverbanks $\mathrm{C}$ and $\mathrm{D}$ ) and the seven bridges (part A is connected to 5 bridges, parts B, C and D are each connected to 3 bridges). This simplified geography of Königsberg can also be represented by a graph, in which the nodes represent the parts of town A-D and the edges represent the bridges.

Relying on this graph-theoretical representation, Euler defines an Euler path as a path through a graph $G$ that includes each edge in $G$ exactly once. Euler uses the notion of an Euler path to reformulate the explanandum in terms of the question: why has everyone failed to traverse Königsberg on an Euler path? His answer to this why-question has two components. 
First, Euler's theorem according to which there is an Euler path through a graph $G$ iff $G$ is an Eulerian graph. Euler proved that a graph $G$ is Eularian iff (i) all the nodes in $\mathrm{G}$ are connected to an even number of edges, or (ii) exactly two nodes in $\mathrm{G}$ (one of which we take as our starting point) are connected to an odd number of edges. ${ }^{10}$

Second, the actual bridges and parts of Königsberg are not isomorphic to an Eulerian graph, because conditions (i) and (ii) in the definition of an Eulerian graph are not satisfied: no part of town (corresponding to the nodes) is connected to an even number of bridges (corresponding to the edges), violating condition (i); and more than two parts of town (corresponding to the nodes) are connected to an odd number of bridges (corresponding to the edges), violating condition (ii). Königsberg could have been isomorphic to an Eulerian graph in 1736, but as a matter of contingent fact it was not.

Therefore, Euler concludes from the first and the second component that there is no Euler path through the actual Königsberg. This explains why nobody ever succeeded in crossing all of the bridges of Königsberg exactly once.

Does the CTE capture Euler's explanation? Euler's explanation has the structure demanded by the CTE: the explanans consists of Euler's theorem (a mathematical and intuitively non-causal generalization) and the statement that all parts are actually connected to an odd number of bridges. The explanandum phenomenon is that everyone has failed to cross the city on an Euler path.

Moreover, all three conditions that the CTE imposes on the explanans and the

\footnotetext{
${ }^{10}$ One may, of course, also ask whether the proof for Euler's theorem is an explanatory proof. However, this question changes the topic and, hence, the explanandum, because it concerns an explanation in pure mathematics (as opposed to an applied mathematical explanation). As mentioned in Section 1, I will not address this topic.
} 
explanandum are satisfied:

1. The veridicality condition holds because (a) Euler's theorem, (b) the statement about the contingent fact that each part of Königsberg is actually connected to an odd number of bridges, and (c) the explanandum statement are all true. ${ }^{11}$

2. The implication condition is met, since Euler's theorem together with the statement about the contingent fact entail the explanandum statement.

3. The dependency condition is satisfied, because Euler's theorem supports the counterfactual "if all parts of Königsberg were connected to an even number of bridges, or if exactly two parts of town were connected to an odd number of bridges, then people would not have failed to cross all of the bridges exactly once".

Therefore, I conclude that the CTE applies to Euler's explanation.

\footnotetext{
${ }^{11}$ Although the veridicality condition is met in the case of Euler's explanation, one might worry that it does not hold for all scientific explanation, because (a) many scientific explanations involve idealized assumption, and (b) how-possibly explanations play an important epistemic role in the sciences. Both idealized and how-possibly explanations do not meet the veridicality condition. Regarding idealized explanations, it is, however, often possible to (re)interpret the idealizations in a way that is compatible with the veridicality condition by adopting, for instance, a dispositionalist and minimalist accounts of idealizations (see Cartwright 1989; Hüttemann 2004; Strevens 2008). Regarding how-possibly explanations, I ultimately agree that the veridicality condition has to be rejected, if the CTE is supposed to be an account of both how-possibly and how-actually explanations. However, many prominent accounts of explanations (including Woodward's version of the CTE) are (at least, implicitly) presented as accounts of how-actually explanations. In this vein, I also introduce the CTE as an account of how-actually explanations; this account can, of course, be weakened in the case of how-possibly explanations (see Reutlinger et al. for my approach to how-possibly explanations and toy models).
} 


\section{Applying the CTE to Metaphysics: Loewer's Explanation}

I will now turn to an example of a non-causal explanation from the domain of metaphysics - which I call 'Loewer's explanation' - and explore whether the CTE captures the example.

Barry Loewer has recently argued that Humean accounts of laws of nature provide a metaphysical explanation. According to this explanation, the Humean mosaic of a world metaphysically explains the nomic facts of that world:

On Lewis' account [of laws] the Humean mosaic metaphysically determines the L-laws. It metaphysically explains (or is part of the explanation together with the characterization of a Best Theory) why specific propositions are laws. (Loewer 2012: 131, emphasis added)

To avoid misunderstandings, Loewer's explanation is just meant to be one example of a non-causal explanation from metaphysics. My goal is not to defend Humeanism, but to argue for the claim that the CTE captures at least some metaphysical explanations and, thereby, explicates what makes them explanatory (see below for my remarks on the scope of the CTE in the realm of metaphysical explanations).

Loewer argues that this metaphysical explanation is typical for a larger class of metaphysical explanations that rely on grounding relations (and perhaps other kinds of metaphysical dependence relations such as constitution, supervenience, realization, and so on): 
The relevant kind of metaphysical explanation is one in which a type of fact - say mental facts - is shown to be grounded in or constituted by some other kind of fact - say neurological fact. (ibid.)

In other words, Loewer seems to hold that if neurological facts ground mental facts, then neurological facts metaphysically explain mental facts; and if the Humean mosaic grounds the nomic facts, then the Humean mosaic metaphysically explains the nomic facts. Proponents of grounding tend to agree with Loewer that grounding relations are intimately connected to explanatory power (see, for instance, Rosen 2010; Fine 2012; Schaffer 2009, 2015; Wilson manuscript, Ruben 2012; see Correira and Schnieder 2012b for an overview). To consider one prominent example, Fine claims that "philosophy is often interested in questions of explanation - of what accounts for what - and it is largely through the employment of the notion of ontological ground that such questions are to be pursued.” (Fine 2012: 40)

However, what does it mean to say that grounding is explanatory? Which theory of explanation captures the explanatory power of grounding (or of grounding claims)? Loewer has little to say in response to this question. He draws attention to the fact that (causal and non-causal) scientific explanations and metaphysical explanations differ in important respects:

Metaphysical explanation need not involve laws [of nature, A.R.] and the explanandum and explanans must be co-temporal (if the explanans is a temporal fact or property). (Loewer 2012: 131) 
Scientific explanation of a particular event or fact need not show that it is grounded in a more fundamental event or fact but rather, typically, shows why the event occurred in terms of prior events and laws. (ibid.)

One further difference between metaphysical and scientific explanation is that the latter but not the former may be probabilistic. (ibid.)

For present purposes, I agree with Loewer that scientific explanations differ from metaphysical explanations with respect to these features.

My focus is on the question whether we can make sense of the explanatory character of metaphysical and scientific explanations by using the same theory of explanation - even if scientific and metaphysical explanation differ in some features, as Loewer claims. Loewer explicitly puts this question aside, since he pursues other goals in his paper: "I don't have anything like a thorough account of metaphysical explanation (or for that matter of scientific explanation)" (Loewer 2012: 131). In the remainder of this section, I will explore whether the CTE captures Loewer's explanation as a specific non-scientific kind of non-causal explanation. My claim is that the CTE applies, if one is prepared to accept a salient epistemological difference between metaphysical and scientific explanations.

Loewer's explanation satisfies the structure that the CTE requires. The explanans consists of two elements: first, a metaphysical and intuitively noncausal grounding generalization 'the entire Humean mosaic grounds the nomic 
facts'. ${ }^{12}$ According to the CTE, grounding generalizations figuring in metaphysical explanations play a similar role to laws of nature in scientific explanations: most importantly, both grounding generalizations and laws of nature license inferences about the explanandum (implication condition of the CTE) and support counterfactuals (dependency condition of the CTE) (see Wilson manuscript). Second, the explanans also includes the auxiliary statements including (i) a description of the entire Humean mosaic $M$ of some world w (in terms of fundamental predicates) ${ }^{13}$, (ii) the assumption that the best system account (BSA) of laws is true (see Lewis 1973, 1994; for Loewer's amendment of Lewis' original account see Loewer 2001, 2008, 2012). The explanandum phenomenon is that certain facts or propositions (call them 'n-facts') are the nomic facts or propositions of a world w. ${ }^{14}$

Does Loewer's explanation satisfy the three constraints of the CTE? Two of the three conditions are met:

- Implication Condition: The elements of the explanans (consisting of the

\footnotetext{
${ }^{12}$ It is a controversial issue whether the Humean grounding generalization (or a Humean supervenience claim) holds in all possible worlds, or only in a restricted class of worlds (see Hall 2012 for an overview).

${ }^{13}$ See Cohen and Callender (2009) for a version of the BSA that is not committed to fundamental (or natural) predicates.

${ }^{14}$ While Loewer seems to accept the grounding generalization as a key part of the explanans, there might be an alternative version of the Humean metaphysical explanation of laws that does not rely on grounding. This version is inspired by the Canberra plan. According to the Canberra plan version, the explanans consists of the following assumptions: (1) Conceptual analysis: A general statement $l$ is a law in world w iff $l$ is a theorem or axiom of the best system of w. (2) Empirical fact: The mosaic of a world $\mathrm{w}$ is such that $l$ is an axiom or theorem of the best system of w. Assumptions (1) and (2) entail the explanandum: (3) Statement $l$ is a law in w. The crucial point here is that the CTE also captures the Canberra plan version of the Humean explanation of laws. Most importantly, the decisive counterfactual is true in Loewer's version and in the Canberra plan version; see below.
} 
description of the entire mosaic $\mathrm{M}$ of world $\mathrm{w}$, the grounding generalization, and the BSA) entail that the $\mathrm{n}$-facts are the nomic facts of $\mathrm{w}$.

- Dependency Condition: The grounding generalization (in conjunction with the BSA) supports a counterfactual of the form: there is at least one metaphysically possible way the mosaic could be, $M^{*}$, such that if the mosaic of world w were $\mathrm{M}^{*}$ (and not $\mathrm{M}$, as it actually is), then the $\mathrm{n}$-facts would not be the laws of $\mathrm{w}$. - Being primitively asymmetric, the grounding relation determines the relevant direction of counterfactual dependence, and, hence, the direction of explanation runs from the Humean mosaic (the grounding fact) to the nomic facts (the grounded facts), and not vice versa. In section 5, I will return to the issue of the asymmetry of non-causal explanations.

Applying the CTE is somewhat less straightforward with regard to the Veridicality Condition. Certainly, Humeans take all of the explanatory assumptions to be true, and these assumptions may as well be true. But we do not know whether the Humean explanans is true. For instance, we do not know what the entire mosaic of our world is like and we do not know whether the Humean grounding generalization is true. Moreover, we do not actually know how to infer the laws from the mosaic; rather we know how to draw this inference only in principle but not in practice (Loewer 2008: 160). Instead of empirical evidence, Humeans rely on indirect epistemological arguments (see, Earman and Roberts 2005) and ontological arguments based on criteria such as parsimony and fruitfulness (a great example is the interchange between the Humean Loewer [Loewer 2012: sect. 5] and the anti-Humean Maudlin [Maudlin 2007: chap. 6]). However, this epistemic situation is by no means a unique feature of Humean 
metaphysics. We also do not know whether the explanatory assumptions of, say, anti-Humeans are true. In fact, this seems to be the normal epistemic situation in metaphysics and an explicitly acknowledged methodological issue in metametaphysics (see, for instance, the contributions in Ross et al. 2013).

This is not necessarily a problem as such, because the CTE, in principle, allows for (scientific and metaphysical) explanations whose explanantia we currently do not (fully) know to be true and perhaps never will - i.e. how-possibly explanations. ${ }^{15}$ Accordingly, one tempting reaction is to interpret metaphysical explanations as how-possibly explanations (Nozick 1981: 8-11). It is quite plausible to interpret a Humean as answering the following how-possibly question: how is it possible that there are laws (and causes and probabilities) without positing 'necessary connections' in nature? Moreover, Loewer's own work in metaphysics of science is a perfect guide to additional how-possibly questions that one might want to ask from a Humean perspective such as: how is it possible that there are causes if the world is fundamentally non-causal (Loewer 2008)? And how is it possible that there are objective probabilities if the world's fundamental dynamical laws are deterministic (Loewer 2001, 2012)? Being concerned with how-possibly questions is nothing to be ashamed of and not unique to metaphysics, because appealing to how-possibly explanations is also a respectable part of science (see Grüne-Yanoff 2007; Weisberg 2013; Reutlinger et al. forthcoming).

To sum up this discussion, the CTE applies to Loewer's metaphysical explanation, if one is willing to relax the veridicality condition and, thereby, allows for how-possibly explanations.

\footnotetext{
${ }^{15}$ See footnote 11 regarding two qualifications with respect to the veridicality condition in the context of scientific explanations.
} 
I want to conclude this section with two remarks about the scope of the CTE in the context of metaphysical explanations.

First, the applicability of the CTE is likely to generalize to Humean metaphysical explanations of causes and objective probabilities - that is, if the Humean mosaic ground facts about causes and probabilities, then the mosaic metaphysically explains what the causes and probabilities in a world are. As highlighted above, I take Humeanism - and Loewer's explanation - to be no more than an illustrative example for a non-causal and also non-scientific explanation. Prima facie, the CTE also applies to anti-Humean metaphysical explanations. For instance, a necessitarian (such as Armstrong 1983) and a primitivist about laws (such as Maudlin 2007) may assert that the laws (that is, a necessitation relation holding between universals, and, respectively, the fundamental laws of temporal evolution) metaphysically explain the existence of regularities in the world. If the CTE is right, then such anti-Humean metaphysical explanations of regularities rely on counterfactuals of the form 'if the laws were different, then the regularities would be different'. In sum, I believe it is only mildly speculative to assert that CTE generalizes to all grounding explanations, if the explanatory grounding facts exist as a matter of metaphysical contingency. For Humeans, the grounding fact, the mosaic, exists contingently; for necessitarians and primitivists, the grounding facts, the laws, exist contingently (for a remark on dispositional essentialism, see footnote 16 below).

Second, the CTE also seems to have clear limitations: prima facie, it does not apply to those metaphysical explanations referring to grounding facts that exist necessarily. Consider a toy example: the mathematical fact that $1+1=2$ (that we take to be a necessary fact) grounds the truth of the sentence ' $1+1=2$ '. Suppose 
that, due to the grounding relation, the mathematical fact that $1+1=2$ metaphysically explains the truth of the sentence ' $1+1=2$ '. According to the CTE, this explanation must involve the counterfactual "if it were the case that $1+1 \neq 2$, then the sentence ' $1+1=2$ ' would be false". The trouble with this counterfactual is that its antecedent expresses an impossible proposition. ${ }^{16}$ In order to deal with this difficulty, a proponent of the CTE faces, at least, three options: (i) to defend the ambitious claim that one can evaluate counterfactuals with (logically or metaphysically) impossible antecedents as non-vacuously true or false, and, moreover, that the truth value of those counterfactuals corresponds to our intuitions about the explanation at hand (see Schaffer 2015: sect. 3.1; also Wilson manuscript), (ii) to deny that the grounding claim in question is explanatory, and (iii) to accept that the CTE does not capture all metaphysical explanations (but only those referring to contingently existing grounding facts), and, hence, full fledged monism fails and the CTE ought to be supplemented by an additional theory of those metaphysical explanations drawing on necessarily true propositions. Discussing the merit of these options is a challenge for future research. I will not even attempt to address these difficult questions in the present

\footnotetext{
${ }^{16}$ At first glance, the same problem appears to arise in the context of dispositional essentialism about laws. According to dispositional essentialism, the nomic essence of some property $\mathrm{P}$ grounds the regularities involving instantiation of $\mathrm{P}$ in a world (see, for instance, Bird 2007). A proponent of the CTE might be tempted to cash out the explanatory content of this grounding claim in terms of the counterfactual "if $\mathrm{P}$ had a non-actual nomic essence, then regularities would be different than they actually are". According to dispositional essentialism, this antecedent is metaphysically impossible. However, a dispositional essentialist is certainly not committed to this prima facie problematic counterfactual within the CTE framework. A much more plausible and more useful counterfactual is this one: 'if some non-actual 'alien' property $\mathrm{P}^{*}$ with a different nomic essence than $\mathrm{P}$ 's nomic essence were instantiated (rather than $\mathrm{P}$ ) in world $\mathrm{w}$, then the regularities in w would be different than the actual regularities'. The antecedent of this conditional is not impossible and suffices to spell out the explanatory content of the grounding claim that the dispositional essentialist makes.
} 
paper. $^{17}$

\section{Qualifications}

Let me add three qualifications in order to sharpen the CTE and to avoid potential misunderstandings.

First, one can still distinguish between causal and non-causal explanations within the CTE framework as follows: non-causal explanations are explanatory by virtue of exhibiting non-causal counterfactual dependencies; causal explanations are explanatory by virtue of exhibiting causal counterfactual dependencies. Although I consider the distinction between causal/non-causal counterfactual dependencies as a primitive for present concerns, I have argued explicitly for a positive account of how one might draw the distinction (see AUTHOR a,b). Following Bertrand Russell (1912/13) and present-day Neo-Russellians, I propose that causal relations are characterized by necessary criteria such as asymmetry, time-asymmetry, distinctness of the relata, metaphysical contingency, and so on. Taking into account the Russellian criteria, my proposal is this: causal explanations reveal causal counterfactual dependencies if the dependency relations instantiate all of the Russellian criteria. Non-causal explanations exhibit non-causal counterfactual dependencies, if the dependency relations do not instantiating all of the Russellian criteria. However, the success of the CTE does not depend on my particular proposal for drawing the causal/non-causal line.

Second, the dependency condition can, to a certain extent, be disentangled

\footnotetext{
${ }^{17}$ I believe the very same problem arises in the context of explanations in pure mathematics, where the assumptions in the explanans seem to include necessary truth. It is an open research questions for me whether the CTE applies to explanations in pure mathematics or whether the CTE needs to be supplemented by an alternative theory of explanations in pure mathematics.
} 
from (I) an interventionist account of causation (in the context of causal explanations), and from (II) a causal interpretation (in the context of non-causal explanations). Let me explain the claims (I) and (II) in detail.

I will turn to claim (I) first. In the context of causal explanations, Woodward interprets the dependency condition of the CTE in terms of interventionist counterfactuals whose antecedents state "if there were a possible intervention on the initial conditions (that is, the purported cause)". However, a proponent of the CTE is not committed to an interventionist account of causation. Other broadly counterfactual accounts are also compatible with the CTE (that is, broadly counterfactual accounts including, for instance, von Wright 1971; Menzies and Price 1993; Lewis 2004; AUTHOR). ${ }^{18}$ In this paper, it is not my goal to argue for any particular counterfactual account of causation. As a consequence, I will not commit myself to the claim that - in the context of causal explanation - the counterfactuals mentioned in the dependency condition of the CTE have to be understood as interventionist counterfactuals (unlike, for instance, Schaffer [2015] and Wilson [manuscript] who defend an interventionist approach to grounding).

Let me now address claim (II), i.e. the claim that the dependency condition can be disentangled from a causal interpretation. When discussing examples of non-causal explanations, Woodward himself voices another prima facie convincing reason for not requiring that all explanatory counterfactuals have the form of interventionist counterfactuals:

18 One reason for choosing a non-interventionist counterfactual account of causation is that Woodward's critics have recently argued that interventionist counterfactuals are inherently problematic and ultimately dispensable for understanding causation and causal explanation (see Strevens 2008). 
"When a theory or derivation answers a what-if-things-had-been different question but we cannot interpret this as an answer to a question about what would happen under an intervention, we may have a non-causal explanation of some sort." (Woodward 2003: 221)

As I understand this quote, Woodward does not require that all explanatory counterfactuals have the form of interventionist counterfactuals: while causal explanations rely on interventionist counterfactuals, there are also non-causal explanations making use of non-interventionist counterfactuals. (Again, for proponents of a non-interventionist counterfactual theory of causation, I propose to draw a distinction between causal and non-causal counterfactuals in terms of the Russellian criteria; see above).

Based on the claims (I) and (II), I work with the minimal assumption that, in the dependency condition of the CTE, one should generally rely on counterfactuals of the form "if $S_{1}, \ldots, S_{n}$ had been different than they actually are (in at least one way deemed possible in the light of the generalizations), then $\mathrm{E}$ or the conditional probability of E would have been different as well". ${ }^{19}$ Furthermore, there is also a positive analogy to Woodward's causal CTE regarding the existentially quantified form of the counterfactuals: the qualification 'in at least one way deemed possible in the light of the generalizations' is analogous to the interventionist requirement that there be some possible intervention on the antecedent variable that leads to a change in the consequent variable; it is not required that all possible interventions have such an effect.

Third, I anticipate a potential worry at this point. One may wonder

${ }^{19}$ In Reutlinger (2013), I provide an in-depth discussion of non-interventionist counterfactuals and their semantics. 
whether the non-causal version of the CTE avoids the problems of the coveringlaw account. I can only sketch how the non-causal version of the CTE responds to these problems:

The problem of irrelevance: To avoid counterexamples such as the birthcontrol pills scenario (Salmon 1989: 50), the non-causal CTE distinguishes between explanatorily relevant and irrelevant factors as follows: a factor is relevant only if the explanandum counterfactually depends on it (dependency condition), otherwise it is an irrelevant factor.

The problem of low probabilities: To deal with the syphilis-paresis scenario (Salmon 1989: 49), the non-causal CTE allows for low probability explanations (implication condition).

The flagpole-shadow scenario: consider the flagpole-shadow scenario (Salmon 1989: 47). The guiding intuition of the flagpole-shadow scenario is that the 'correct' explanation is a causal explanation (the height of the flagpole causally explains the length of the shadow) and that the covering law account does not have the means to identify it. One might worry that the CTE is also unable to identify the 'correct' causal explanation underlying the flagpole scenario. This worry is, however, unjustified, because the CTE is a theory of causal and non-causal explanations. Applying the CTE to causal explanations may take the form of supplementing the CTE with Woodward's interventionist account of causation as the underlying theory of causation. As Woodward argues successfully (to my mind), a causal version of the CTE adequately describes the flagpole scenario (Woodward 2003: 155, 361). However, as I pointed out in the second qualification above, a proponent of the CTE is not committed to Woodward's interventionist theory of causation. The CTE in combination with 
other counterfactual accounts of causation (that do not rely on interventions) also correctly identifies the causal structure underlying the flagpole-shadow scenario (such counterfactual accounts of causation include, for instance, von Wright 1971; Lewis' 2004; Menzies and Price 1993; AUTHOR). Thus, although the flagpoleshadow scenario is a counterexample to the covering-law accounts, it is not a threat to the CTE.

With respect to the flagpole-shadow scenario ${ }^{20}$, let me emphasize that one should disentangle two questions: (1) the question whether the flagpole-shadow scenario is a counterexample to the CTE, and (2) the question whether all noncausal explanation display an asymmetry. The answers to these questions are logically independent of one another. Regarding (1), I hold that the flagpoleshadow scenario is not a counter-example to the CTE (as I argued above). Regarding (2), I think there are good reasons to hold that some non-causal explanations are not asymmetric. ${ }^{21}$ While I agree that all causal explanations are asymmetric, I believe that some (for instance, Euler's explanation), but not necessarily all, non-causal explanations lack such an asymmetry in that the counterfactual dependence in question is symmetric (for an elaborate argument for this claim see Frisch and Reutlinger manuscript; Kistler 2013).

However, the situation is importantly different and more straightforward for metaphysical grounding explanations. The grounding relation underlying a metaphysical explanation is usually taken to be (primitively) asymmetric (Fine 2012; Schaffer 2015; Schnieder forthcoming; see Correira and Schnieder 2012b for an overview). Given that the grounding relation has an in-built asymmetry, the

\footnotetext{
${ }^{20}$ I would like to thank a referee for urging me to address this point.

${ }^{21}$ This claim is not necessarily at odds with recent attempts to justify the claim that some non-causal explanations do in fact display an asymmetry (see Lange 2011; Jansson forthcoming).
} 
asymmetry of metaphysical explanations mirrors the underlying asymmetry of grounding (just as the directionality of some scientific explanations mirrors the causal asymmetry). Although I accept that the asymmetry is primitively built into the grounding relation, I do not claim that the explanatory character of grounding relations is a primitively built-in feature of this relation. Instead, the main claim of my paper is that the CTE provides an explication of the explanatory character of grounding explanations (see Section 4).

\section{Conclusion}

In the recent philosophy of explanation, a consensus has emerged, according to which there are non-causal explanations in the natural and social sciences, in pure mathematics, and in metaphysics - in addition to familiar causal explanations in the sciences. I have argued for the claim that the counterfactual theory of explanation (CTE) captures the explanatory character of scientific and metaphysical explanations. According to the CTE, causal and non-causal explanations are explanatory by virtue of revealing counterfactual dependencies between the explanandum and the explanans. I have argued that the CTE is applicable to two paradigms of non-causal explanations in the two areas of interest: Euler's explanation (a toy example of a non-causal scientific explanation) and Loewer's explanation (an example of a non-causal metaphysical explanation), if one allows for a relaxation of the veridicality condition. For this reason, I believe that the CTE is a promising monist approach to explanations in science and metaphysics alike. 


\section{References}

Achinstein, P. (1983): The Nature of Explanation. New York: Oxford University Press.

Armstrong, D. (1983): What Is A Law of Nature?, Cambridge, UK: Cambridge University Press.

Barabasi, A., Newman, M., Watts, D. (2006): The Structure and Dynamics of Networks, Princeton: Princeton University Press.

Batterman, R. (2000): "Multiple Realizability and Universality." British Journal for Philosophy of Science 51: 115-145.

Batterman, R. (2002): The Devil in the Details, New York: Oxford University Press.

Bechtel, W. and Richardson, R. (1993): Discovering Complexity. Princeton: Princeton University Press.

Bird, A. (2007): Nature's Metaphysics, Oxford: Oxford University Press.

Bokulich, A. (2008): “Can Classical Structures Explain Quantum Phenomena?” British Journal for the Philosophy of Science 59(2): 217-235.

Bueno, O. and French, S. (2012): "Can Mathematics Explain Physical Phenomena?", British Journal for the Philosophy of Science 63: 85-113.

Cartwright, N. (1989): Nature's Capacities and Their Measurement, Oxford: Clarendon Press.

Cohen, J. and Callender, C. (2009): “A Better Best System Account of Lawhood”, Philosophical Studies 145: 1-34.

Correira, F. and B. Schnieder (eds.) (2012a): Metaphysical Grounding: Understanding the structure of reality, Cambridge: Cambridge University 
Press.

Correira, F. and B. Schnieder (2012b): "Grounding: An Opinionated Introduction", In Metaphysical Grounding: Understanding the structure of reality, Correira, F. and B. Schnieder (eds.), pp. 1-36, Cambridge: Cambridge University Press.

Earman, J. and Roberts, J. (2005): "Contact with the Nomic. A Challenge for Deniers of Humean Supervenience about Laws of Nature Part II: The Epistemological Argument for Humean Supervenience", Philosophy and Phenomenological Research LXXI: 253-286.

Fine, K. (2001): “The question of realism”, Philosophers' Imprint 1: 1-30.

Fine, K. (2012): “Guide to ground”, In F. Correia and B. Schnieder (Eds.), Metaphysical grounding: Understanding the structure of reality (pp. 3780). Cambridge: Cambridge University Press.

Frisch, M. (1998): Theories, Models, and Explanation, Dissertation, UC Berkeley. Frisch, M. and Reutlinger, A. (manuscript): “Are All Non-Causal Explanations Asymmetric?”.

Hall, N. (2012): “David Lewis's Metaphysics", The Stanford Encyclopedia of Philosophy (Fall 2012 Edition), Edward N. Zalta(ed.), URL = $<$ http://plato.stanford.edu/archives/fall2012/entries/lewis-metaphysics/>.

Hempel, C. G. (1965): Aspects of Scientific Explanation, New York: Free Press.

Huneman, P. (2010): “Topological Explanations and Robustness in Biological Sciences" Synthese 177: 213-245.

Hüttemann, A. (2004): What's Wrong With Microphysicalism?, London: Routledge.

Jansson, L. (forthcoming): "Explanatory Asymmetries: Laws of Nature 
Rehabilitated", Journal of Philosophy.

Kim, J. (1974): "Noncausal Connections", Noûs 8 (1): 41-52.

Kistler, M. (2012): “The Interventionist Account of Causation and Non-Causal Association Laws", Erkenntnis 78: 1-20.

Kitcher, P. (1984): The Nature of Mathematical Knowledge. Oxford: Oxford University Press.

Kitcher, P. (1989): "Explanatory Unification and the Causal Structure of the World." In Minnesota Studies in the Philosophy of Science. Vol. 13, Scientific Explanation, ed. P. Kitcher and W. Salmon, 410-505. Minneapolis: University of Minnesota Press.

Lange, M. (2009a): “Dimensional Explanations.” Noûs 43: 742-775.

Lange, M. (2009b): "Why do the Laws Explain Why?", In T. Handfield (ed.) Dispositions and Causes, pp. 286-321, New York: Oxford University Press.

Lange, M. (2011): “Conservation Laws in Scientific Explanations: Constraints or Coincidences?.” Philosophy of Science 78: 333-352.

Lange, M. (2013a): "What Makes a Scientific Explanation Distinctively Mathematical?." British Journal for the Philosophy of Science 64: 485-511. Lange, M. (2013b): "Really Statistical Explanations and Genetic Drift." Philosophy of Science 80: 169-88.

Lange, M. (2014): “Aspects of Mathematical Explanation: Symmetry, Unity, and Salience”, Philosophical Review, 123.4 (2014): 485-531.

Lewis, D. (1973): Counterfactuals, Oxford: Oxford University Press.

Lewis, D. (1986): “Causal Explanation.” In D. Lewis (1986) Philosophical Papers Vol. II, New York: Oxford University Press, 214-240. 
Lewis, D. (1994): "Humean Supervenience Debugged", in: D. Lewis, Papers in Metaphysics and Epistemology, Cambridge: Cambridge University Press, pp. 224-247.

Lewis, D. (2004): "Causation as Influence", in J. Collins, N. Hall and L.A. Paul (eds.), Causation and Counterfactuals, Cambridge, MA: MIT Press, 75-106.

Lipton, P. (2004): Inference to the Best Explanation, Second Edition, London: Routledge.

Loewer, B. (2001): "Determinism and Chance", Studies in the History and Philosophy of Modern Physics 32: 609-620.

Loewer, B. (2008): "Why there is anything except physics", In J. Hohwy and J. Kallestrup (eds.), Being Reduced. New essays on reduction, explanation, and causation (pp. 149-163). Oxford: Oxford University Press.

Loewer, B. (2012): “Two Accounts of Laws and Time", Philosophical Studies 160: $115-137$.

Machamer, P., L. Darden, and C. Craver (2000): “Thinking about Mechamisms," Philosophy of Science 67: 1-25.

Mancosu, P. (2008): “Mathematical Explanation: Why it matters”, in P. Mancosu, ed., The Philosophy of Mathematical Practice, Oxford: Oxford University Press, 134-150.

Mancosu, P. (2011): "Explanation in Mathematics" The Stanford Encyclopedia of Philosophy.

Maudlin, T., (2007): The Metaphysics Within Physics, New York: Oxford University Press.

Menzies, P. and H. Price (1993): "Causation as a Secondary Quality", British 
Journal of the Philosophy of Science, 44: 187-203.

Nozick, R. (1981): Philosophical Explanations. Cambridge, MA: Harvard University Press.

Pexton, M. (2014): “How Dimensional Analysis Can Explain”, Synthese, DOI: 10.1007/s11229-014-0401-x.

Pincock, C. (2015): “Abstract Explanations in Science”, British Journal for Philosophy of Science 66: 857-882

Pincock, C. (2012): Mathematics and Scientific Representation, New York: Oxford University Press.

Reutlinger, A. (2013): A Theory of Causation in the Biological and Social Sciences, New York: Palgrave Macmillan.

Reutlinger, A. (forthcoming): "Is There A Monist Theory of Causal and NonCausal Explanations? The Counterfactual Theory of Scientific Explanation", Philosophy of Science.

Reutlinger, A., Hangleiter, D., Hartmann, S. (forthcoming): “Understanding (with) Toy Models", British Journal for Philosophy of Science.

Rosen, G. (2010): “Metaphysical dependence: Grounding and reduction." In B. Hale and A. Hoffmann (Eds.), Modality: Metaphysics, logic, and epistemology (pp. 109-136). Oxford: Oxford University Press.

Ross, D., Ladyman, J. and H. Kincaid (2013): Scientific Metaphysics, New York: Oxford University Press.

Ruben, D.-H. (2012) Explaining Explanation, Boulder: Paradigm Publishers.

Russell, B. (1912/13): “On the Notion of Cause.” Proceedings of the Aristotelian Society 13: 1-26.

Salmon, W. (1984): Scientific Explanation and the Causal Structure of the World, 
Princeton: Princeton University Press.

Salmon, W. (1989): Four Decades of Scientific Explanation. Pittsburgh, PA: University of Pittsburgh Press.

Saatsi, J. (forthcoming): "The Geometry of Motion." British Journal for Philosophy of Science.

Saatsi, J. and M. Pexton (2013): "Reassessing Woodward's account of explanation: regularities, counterfactuals, and non-causal explanations." Philosophy of Science 80: 613-624.

Schaffer, J. (2009): On what grounds what. In D. Chalmers, D. Manley, and R. Wasserman (Eds.), Metametaphysics: New essays on the foundations of ontology (pp. 347-383). Oxford: Oxford University Press.

Schaffer, J. (2015): "Grounding in the Image of Causation", Philosophical Studies, Online First: DOI 10.1007/s11098-014-0438-1.

Schnieder, B. (2011): “A Logic for 'Because”, Review of Symbolic Logic 4(3), 445-465.

Schnieder, B. (forthcoming): "The Asymmetry of 'Because", Grazer Philosophische Studien.

Skow, B. (2014): “Are there non-causal explanations (of particular events)?.” British Journal for the Philosophy of Science 65: 445-467.

Steiner, Mark. (1978a): "Mathematical Explanation.” Philosophical Studies 34: $135-151$.

Strevens, M. (forthcoming): “The Mathematical Route to Causal Understanding”, Explanation Beyond Causation, Alexander Reutlinger and Juha Saatsi, Oxford: Oxford University Press.

Strevens, M. (2007): "Review of Woodward, Making Things Happen." 
Philosophy and Phenomenological Research 74(1): 233-49.

Strevens, M. (2008): Depth. Cambridge, MA: Harvard University Press.

Strogatz, S. (1994): Nonlinear Dynamics and Chaos, Cambridge: Westview Press.

Van Fraassen, B. (1980): The Scientific Image. Oxford: Clarendon Press.

Van Fraassen, B. (1989): Laws and Symmetries, Oxford: Clarendon Press.

Von Wright, G.H. (1971): Explanation and Understanding, Ithaca/London: Cornell University Press.

Weatherall, J. (2011): “On (Some) Explanations in Physics." Philosophy of Science 78: 421-447.

Wilson, A. (manuscript): "Metaphysical Causation".

Woodward, J. (1979): "Scientific Explanation”, British Journal for Philosophy of Science 30: 41-67.

Woodward, J. (2003): Making Things Happen. New York: Oxford University Press.

Woodward, J. and C. Hitchcock (2003): “Explanatory Generalizations, Part I: A Counterfactual Account." Noûs 37: 1-24. 